\title{
In-gap states of magnetic impurity in quantum spin Hall insulator proximitized to a superconductor
}

\author{
Szczepan Głodzik ${ }^{1, *}$ and Tadeusz Domański ${ }^{1, \dagger}$ \\ ${ }^{1}$ Institute of Physics, M. Curie-Sktodowska University, 20-031 Lublin, Poland
}

(Dated: August 12, 2020)

\begin{abstract}
We study in-gap states of a single magnetic impurity embedded in a honeycomb monolayer which is deposited on superconducting substrate. The intrinsic spin-orbit coupling induces the quantum spin Hall insulating (QSHI) phase gapped around the Fermi energy. Under such circumstances we consider the emergence of Shiba-like bound states driven by the superconducting proximity effect. We investigate their topography, spin-polarization and signatures of the quantum phase transition manifested by reversal of the local currents circulating around the magnetic impurity. These phenomena might be important for more exotic in-gap quasiparticles in such complex nanostructures as magnetic nanowires or islands, where the spin-orbit interaction along with the proximity induced electron pairing give rise to topological phases hosting the protected boundary modes.
\end{abstract}

\section{INTRODUCTION}

Even a tiny content of impurities introduced to insulating and semiconducting materials can tremendously affect their charge transport, contributing particle/hole carriers from the donor/acceptor level to the conduction/valence band. This is in contrast with completely opposite (destructive) effect played by the magnetic impurities in superconductors where they break the Cooper pairs, leading to formation of the bound Yu-ShibaRusinov (YSR) or briefly Shiba states inside the energy gap $^{1}$. These in-gap states can eventually activate the charge transport in interfaces and heterostructures, owing to the anomalous particle-to-hole (or hole-to-particle) Andreev scattering mechanism ${ }^{2}$. In all such cases impurities are intimately related with existence of the subgap states, whose nature differs depending on the host material. One may hence ask, whether there can be established any connection between such contrasting in-gap states of insulators and superconductors?

A promising platform for addressing this question could be a graphene sheet deposited on $s$-wave superconducting substrate. Electrons of such carbon atoms layer reveal a number of unique properties. Besides their Dirac-like behavior, stemming simply from a honeycomb geometry, the intrinsic spin-orbit coupling (SOC) can induce the QSHI phase ${ }^{3}$ with the spin currents circulating along its boundaries. Such effect has been experimentally observed in graphene randomly decorated with the dilute $\mathrm{Bi}_{2} \mathrm{Te}_{3}$ nanoparticles ${ }^{4}$ and in a heterostructure, consisting of a monolayer of $\mathrm{WTe}_{2}$ placed between two layers of hexagonal boron nitride which has revealed topological properties up to relatively high temperatures up to $100 \mathrm{~K}^{5}$. Further phenomena related with electron pairing arise when a graphene sheet is proximitized to superconducting material ${ }^{6-11}$. For instance, graphene deposited on aluminum films acquires superconductivity with the effective coherence length $\xi \simeq 400 \mathrm{~nm}^{11}$, whereas grown on rhenium it shows high transparency of the interface, with the induced pairing gap $\Delta=330 \pm 10 \mu \mathrm{eV}^{8}$. Upon introducing impurities into proximitized graphene, there

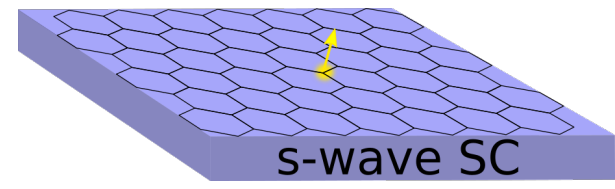

FIG. 1. Scheme of a magnetic impurity embedded in a honeycomb monolayer and proximitized to $s$-wave superconductor.

emerge various in-gap states, manifesting either the topologically trivial or non-trivial phases ${ }^{12}$. Another system for investigating the bound states of magnetic impurities might be possible in bilayer graphene, where upon twisting the carbon sheets to a small 'magic' angle ${ }^{13,14}$ or tuning the interlayer coupling ${ }^{15}$ the intrinsic unconventional superconducting phase is induced.

Here we investigate the properties of in-gap bound states formed at magnetic impurity embedded into the single honeycomb 2-dimensional layer and proximitized to superconductor (Fig. 1), discussing feasible tools to unambiguously distinguish their Shiba-type character in presence of the QSHI phase. This problem has recently gained a great deal of interest, both experimentally ${ }^{16-20}$ and theoretically ${ }^{21-23}$ because similar magnetic structures, e.g. nanowires ${ }^{24,25}$ and nanostripes ${ }^{26,27}$ could enable realization of the Majorana quasiparticles.

The spin-orbit gap in graphene is often claimed to be rather small, although Sichau et al..$^{28}$ have estimated its magnitude (by means of the resistively-detected electron spin resonance) to be $40 \mu \mathrm{eV}$. Under such circumstances the superconducting gap might be comparable to the SOC and this would be sufficient for appearance of the in-gap bound states strictly related with electron pairing. In what follows we perform a systematic study of the Shiba states, inspecting (a) their spatial extent and topography, (b) magnetic polarization, and (c) observable features of the quantum phase $(0-\pi)$ transition manifested by reversal of the orbital currents circulating around the impurity site.

The paper is organized as follows. In Sec. II we introduce the microscopic model and present the method 
for studying the bound states of magnetic impurity existing in honeycomb sheet. Sec. III discusses influence of the insulating and superconducting phases on the ingap quasiparticles and presents their detailed properties. Finally, in Sec. IV, we summarize the results.

\section{MODEL AND METHOD}

We describe the magnetic impurity embedded in a honeycomb sheet (Fig. 1) by the tight-binding Hamiltonian

$$
\hat{H}=\hat{H}_{i m p}+\hat{H}_{K-M}+\hat{H}_{R a s h b a}+\hat{H}_{\text {prox }}
$$

In what follows, this impurity is treated classically

$$
\hat{H}_{i m p}=-J\left(c_{\mathbf{i}_{0} \uparrow}^{\dagger} c_{\mathbf{i}_{0} \uparrow}-c_{\mathbf{i}_{0} \downarrow}^{\dagger} c_{\mathbf{i}_{0} \downarrow}\right)
$$

where we denote the impurity site as $\mathbf{i}_{0}$, and we apply the Kane-Mele scenario ${ }^{3}$ for description of the itinerant electrons

$$
\begin{aligned}
\hat{H}_{K-M} & =-t \sum_{\langle\mathbf{i} \mathbf{j}\rangle \sigma \sigma^{\prime}} c_{\mathbf{i} \sigma}^{\dagger} c_{\mathbf{j} \sigma}-\mu \sum_{\mathbf{i} \sigma} c_{\mathbf{i} \sigma}^{\dagger} c_{\mathbf{i} \sigma} \\
& +i \lambda_{S O} \sum_{\langle\langle\mathbf{i}\rangle\rangle \sigma \sigma^{\prime}} \nu_{\mathbf{i j}} c_{\mathbf{i} \sigma}^{\dagger} s_{z}^{\sigma \sigma^{\prime}} c_{\mathbf{j} \sigma^{\prime}}
\end{aligned}
$$

with the nearest-neighbor hopping $t$, the chemical potential $\mu$ (which we assume to be zero unless otherwise stated), and the imaginary, spin-dependent, next-nearest neighbor hopping amplitude $\lambda_{S O}$. The latter term is responsible for inducing the helical edge states. The sign $\nu_{\mathbf{i j}}= \pm 1$ depends on the direction of electron hopping between the next-nearest-neighbor sites $(+1$ for clockwise and -1 for anticlockwise). The hopping terms involve the summation over (next-)nearest $(\langle\langle\mathbf{i j}\rangle\rangle)\langle\mathbf{i j}\rangle$ neighbors. Since the substrate violates the mirror inversion $z \longrightarrow-z$ symmetry, we also consider the Rashba spin-orbit interaction

$$
\hat{H}_{R a s h b a}=i \lambda_{R} \sum_{\langle\mathbf{i j}\rangle \sigma \sigma^{\prime}} c_{\mathbf{i} \sigma}^{\dagger}\left(\mathbf{s}^{\sigma \sigma^{\prime}} \times \mathbf{d}_{\mathbf{i j}}\right)_{\vec{z}} c_{\mathbf{j} \sigma^{\prime}} .
$$

Here $\mathbf{s}^{\sigma \sigma^{\prime}}$ is the vector of the Pauli matrices, referring to spin $\frac{1}{2}$, and the vector $\mathbf{d}_{\mathbf{i j}}$ connects site $\mathbf{i}$ with its nearest neighbor site $\mathbf{j}$.

Finally, we assume that the honeycomb layer is proximitized to $s$-wave superconductor

$$
\hat{H}_{\text {prox }}=\sum_{\mathbf{i}}\left(\Delta c_{\mathbf{i} \uparrow}^{\dagger} c_{\mathbf{i} \downarrow}^{\dagger}+\text { h.c. }\right),
$$

which induces the on-site pairing $\Delta$. For computing the observables of interest, we perform the BogoliubovValatin transformation

$$
c_{\mathbf{i} \sigma}=\sum_{n}^{\prime}\left(u_{\mathbf{i} \sigma}^{n} \gamma_{n}-\sigma v_{\mathbf{i} \sigma}^{* n} \gamma_{n}^{\dagger}\right),
$$

where' denotes summation over the positive eigenvalues, and numerically solve the equations

$$
\sum_{\mathbf{j}} \hat{H}_{\mathrm{ij}} \hat{\Phi}_{\mathbf{j}}=E_{n} \hat{\Phi}_{\mathbf{i}}
$$

in the auxiliary (Nambu spinor) representation $\Phi_{\mathbf{i}}=$ $\left(u_{\mathbf{i} \uparrow}^{n}, u_{\mathbf{i} \downarrow}^{n}, v_{\mathbf{i} \uparrow}^{n}, v_{\mathbf{i} \downarrow}^{n}\right)^{T}$. The matrix elements read

$$
\hat{H}_{\mathbf{i j}}=\left(\begin{array}{cccc}
\tilde{t}_{\mathbf{i j} \uparrow} & \lambda_{R}^{\uparrow \downarrow} & 0 & \Delta \\
\lambda_{R}^{\downarrow \uparrow} & \tilde{t}_{\mathbf{i j} \downarrow} & \Delta & 0 \\
0 & \Delta^{*} & -\left(\tilde{t}_{\mathbf{i j} \uparrow}\right)^{*} & \left(\lambda_{R}^{\uparrow \downarrow}\right)^{*} \\
\Delta^{*} & 0 & \left(\lambda_{R}^{\downarrow \uparrow}\right)^{*} & -\left(\tilde{t}_{\mathbf{i j} \downarrow}\right)^{*}
\end{array}\right),
$$

where $\tilde{t}_{\mathbf{i j} \sigma}=t_{\mathbf{j}} \delta_{\langle\mathbf{i} \mathbf{j}\rangle}-\left(\mu+\sigma J \delta_{\mathbf{i i}_{\mathbf{o}}}\right) \delta_{\mathbf{i j}}+\sigma i \lambda_{S O} \nu_{\mathbf{j}} \delta_{\langle\langle\mathbf{i} \mathbf{j}\rangle\rangle}$ and $\lambda_{R}^{\sigma \sigma^{\prime}}=i \lambda_{R} \sum_{\sigma \sigma^{\prime}\langle\mathbf{j}\rangle}\left(\mathbf{s}^{\sigma \sigma^{\prime}} \times \mathbf{d}_{\mathbf{i j}}\right)_{\vec{z}}=\left(\lambda_{R}^{\sigma^{\prime} \sigma}\right)^{*}$.

Results discussed in this paper are obtained from numerical diagonalization of the Hamiltonian matrix on $40 \times 40$ lattice with the periodic boundary conditions in both directions. We do not consider any intrinsic pairing mechanism, assuming that it originates solely from the proximity effect (5). Self-consistent treatment of electron pairing is in general important ${ }^{29,30}$, however, in the present case it would not imply any significant changes of the local order parameter ${ }^{31}$.

\section{SUBGAP QUASIPARTICLES}

For a systematic analysis of the subgap quasiparticles we shall start by discussing the in-gap states hosted in the insulating (QSHI) phase and next consider their mutation caused by the electron pairing $\Delta$.

\section{A. Impurity bound states in QSHI phase}

Let us consider the magnetic impurity in a finite-size graphene layer, neglecting the superconducting substrate $(\Delta=0)$. Fig. 2 shows how the intrinsic spin-orbit interaction affects the low-energy quasiparticles. We notice that insulating energy gap of the QSHI phase grows linearly upon increasing the Kane-Mele coupling and, around $\lambda_{S O}=0.2 t$, it saturates to $\sim 1 t$. Concomitantly there appear two in-gap states (purple-dotted lines in Fig. 2), which are fully spin-polarized. Similar bound states have been previously found for a single impurity whose magnetic moment is parallel to the graphene plane $^{32}$. When impurity is close enough to a perimeter of the sample they have been shown to hybridize with the topological edge states, inducing antiresonances in the transmission matrix. It has been also emphasized, that the bound states around point impurity in a two-dimensional insulator could distinguish between the topological and trivial phases of the host material ${ }^{33}$.

Bottom panel in Fig. 2 displays the topography of the occupied $\left(E<E_{F}\right)$ bound state for two different values of 


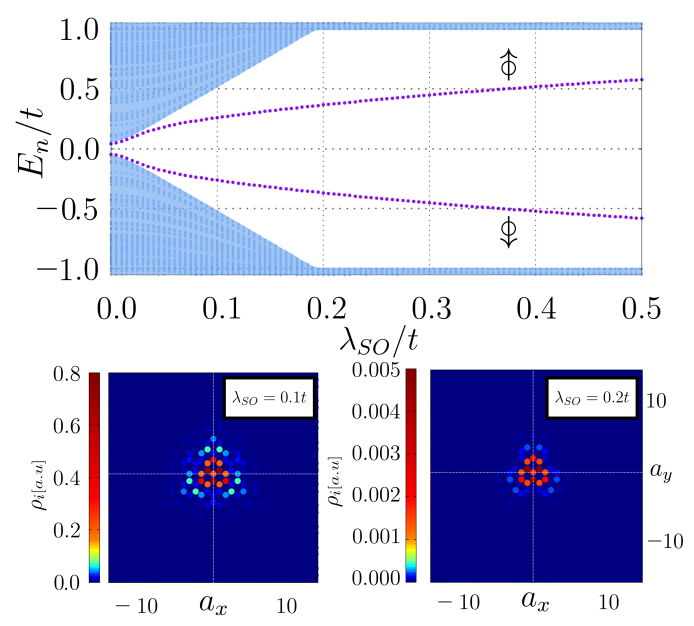

FIG. 2. Low energy spectrum of the honeycomb lattice with the single magnetic impurity as a function of the Kane-Mele coupling $\lambda_{S O}$, assuming $J=6 t$ and $\lambda_{R}=0, \Delta=0$. Bottom panel: Topography of the occupied bound state for $\lambda_{S O}=$ $0.1 t$ and $\lambda_{S O}=0.2 t$.

$\lambda_{S O}$. From careful examination of the spectral weight on the lattice sites adjacent to the impurity, we can notice an oscillatory decay of the wavefunction of the bound state. Practically its spatial extent does not exceed 10 atomic distances, and it quickly vanishes for higher magnitudes of the SOC. This loss of spatial extent is accompanied by the simultaneous reduction of the spectral weight of the bound state. Closely related effects have been previously pointed out for the magnetic ${ }^{34-38}$, non-magnetic ${ }^{12,39,40}$ and both types of the scattering potential as well ${ }^{41-43}$.

\section{B. Shiba quasiparticles}

Upon coupling the honeycomb lattice to superconducting substrate, the energy gap around the Fermi energy results from a combined effect of the proximity induced pairing $(\Delta \neq 0)$ and the insulating phase. In general, these phenomena are known to be competitive as indeed manifested by suppression of the bulk order parameter $\left\langle c_{\mathbf{i} \downarrow} c_{\mathbf{i} \uparrow}\right\rangle$ (Sec. III C). From a perspective of the local physics (at impurity site), however, relationship between the QSHI and superconducting phases is much more intriguing. By gradually increasing the pairing potential $\Delta$, what can be achieved e.g. by reducing the external magnetic field or varying the temperature, we observe development of the Shiba quasiparticles ${ }^{1}$ directly from in-gap quasiparticles of the insulating phase (Fig. 3). Let us remark, that direct transition from the insulating to superconducting phase has been theoretically considered for bulk materials within the mean field ${ }^{44}$ and more sophisticated many-body methods ${ }^{45}$. Such scenario could be practically realized in variety of systems, e.g. thin superconducting films ${ }^{46}$, at oxide interfaces ${ }^{47}$, in organic materials ${ }^{48}$ and possibly in the doped cuprate

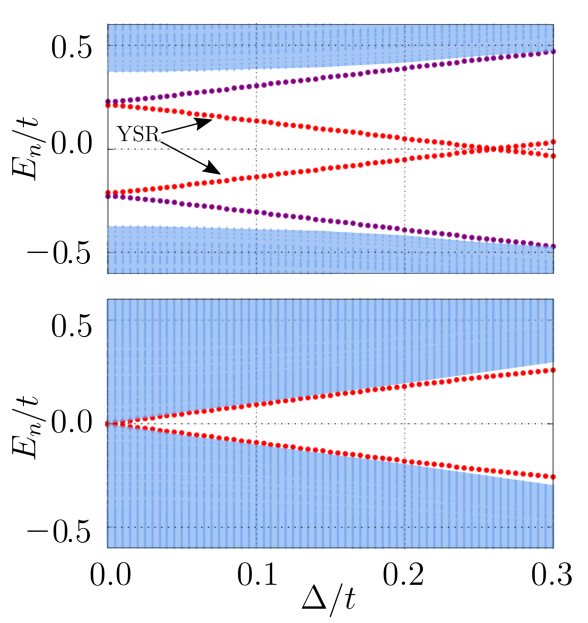

FIG. 3. Top panel: emergence of YSR states (dotted red lines) from in-gap quasiparticles of the QSHI phase (dotted violet lines) driven by the proximity induced pairing $\Delta$ for $J=6 t, \lambda_{S O}=0.1 t, \mu=0$. Bottom panel: same but for $\mu=3 \sqrt{3} \lambda_{S O}$.

superconductors ${ }^{49}$. In the present context we focus on the subgap Shiba-like quasiparticles, which to our knowledge have not been considered so far. To compare our results with less exotic situation, we plot in the bottom panel of Fig. 3 the same situation as in the top panel, but with a value of chemical potential wich is known to close the spin-orbit gap. The system is then metallic and opening the superconducting gap results in a picture similar to traditionally understood Shiba states ${ }^{1}$.

Let us focus in more detail on the Shiba quasiparticles. In the present case they do not obey the original formula $E_{Y S R}= \pm \Delta\left(1-\pi \rho_{n}\left(E_{F}\right) J\right) /\left(1+\pi \rho_{n}\left(E_{F}\right) J\right)$ derived for conventional superconductors because of the vanishing normal density of states in graphene $\rho_{n}\left(E_{F}\right)=0^{50,51}$.

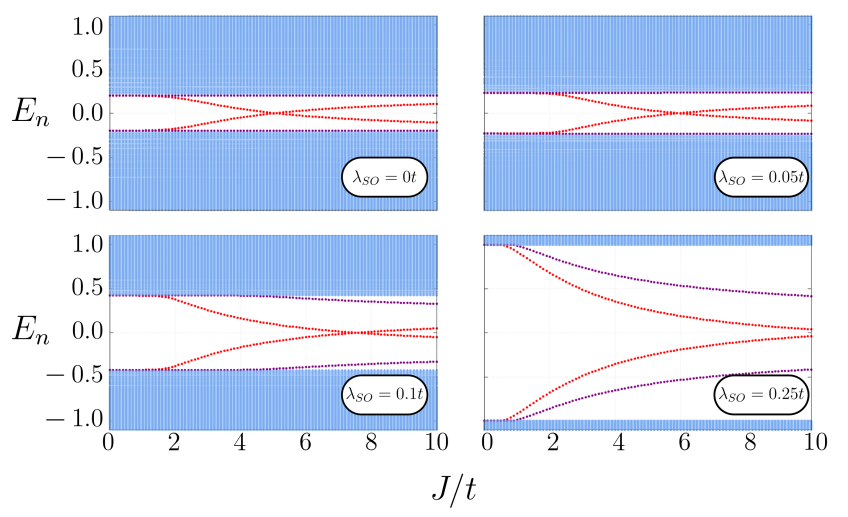

FIG. 4. Evolution of the subgap spectrum with respect to the impurity potential $J$ obtained for $\Delta=0.2 t, \lambda_{R}=0.05 t$ and several values of $\lambda_{S O}$, as indicated. The dotted red and violet lines refer to the YSR states and in-gap quasiparticles of insulating phase, respectively. 

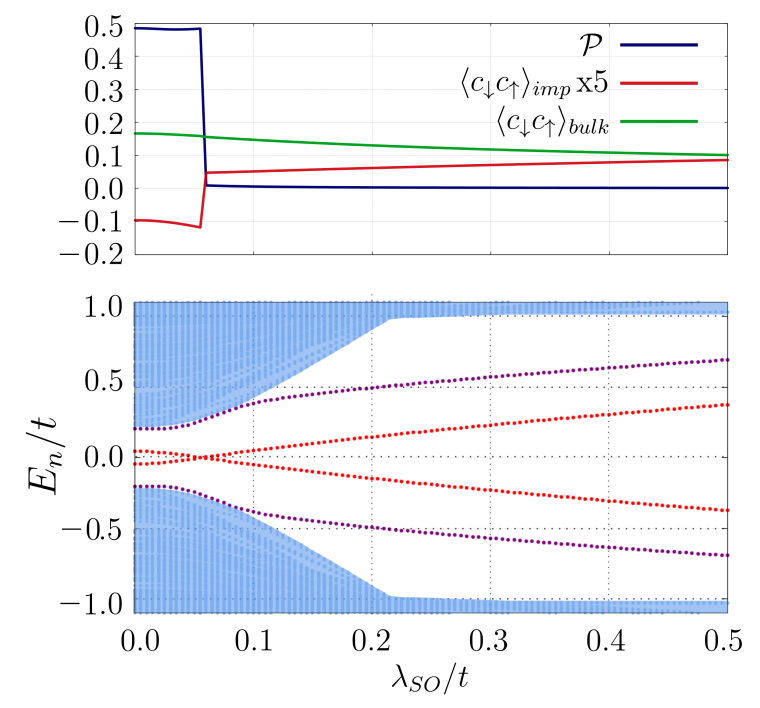

FIG. 5. The polarization $\mathcal{P}$ and order parameter at the impurity site and for the bulk as functions of $\lambda_{S O}$ (top panel). QPT driven at $\lambda_{S O} \simeq 0.05 t$ (bottom panel). Results obtained for the model parameters $\Delta=0.2 t, \lambda_{R}=0.05 t$, and $J=6 t$.

Fig. 4 displays the quasiparticle energies obtained numericaly for our model as a function of the magnetic potential $J$ for several values of Kane-Mele coupling $\lambda_{S O}$. The dense (light-blue) dots refer to a continuum, whereas the single dotted lines represent the in-gap bound states. Amongst these in-gap branches we can recognize the Shiba-like quasiparticles by their strong variation with respect to $J$. In particular, at some critical value $J_{c}$ they eventually cross each other, signaling a qualitative changeover of the ground state ${ }^{52}$. This quantum phase transition (QPT) manifests itself by: sign-reversal of the local order parameter $(0-\pi$ transition $)$, abrupt onset of the spin polarization (Sec. III C), and by qualitative changes (both, in magnitude and vorticity) of the locally circulating currents (Sec. IIIE).

Our analysis indicates, that Kane-Mele coupling $\lambda_{S O}$ affects the QPT, by (i) shifting the critical coupling $J_{C}$ to higher values (Figs 4 and 6) and (ii) leading to substantial changes both in topography and spatial extent of the Shiba-like states (Sec. IIID). Thus the spin-orbit interaction weakens the efficiency of magnetic coupling $J$ between the impurity and conduction electrons. Furthermore, the Shiba states no longer merge with a continuum even in the extremely strong coupling limit $J \rightarrow \infty$, in stark contrast to behavior of magnetic impurities in triangular lattice of the 2-dimensional superconductor ${ }^{21}$ where the Kane-Mele interaction is absent.

\section{QPT}

Let us now focus on the QPT, driven by the intrinsic SOC. Even though variation of $\lambda_{S O}$ would be rather

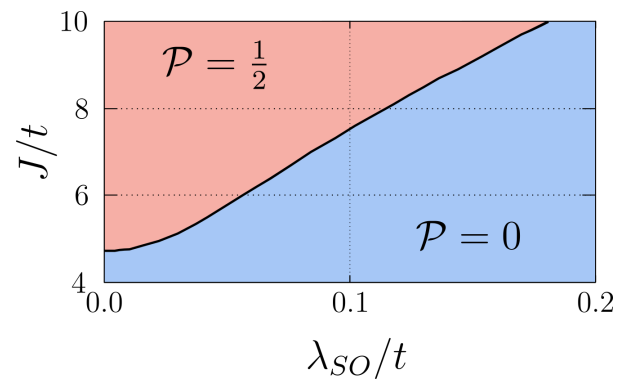

FIG. 6. Variation of the QPT point (corresponding to crossing of the Shiba states) versus the Kane-Mele coupling $\lambda_{S O}$ and impurity potential $J$.

not feasible experimentally, we deem that its effect can be instructive for understanding mutual relationship between the on-site pairing and the spin-orbit interaction. Bottom panel of Fig. 5 presents the eigenenergies, corresponding to the same set of model parameters as in Fig. 2 but in presence of finite $\Delta$ and $\lambda_{R}$. We observe that energy gap of superconducting states $(\sim 0.2 t)$ gradually evolves into the gap of QSHI which saturates around $\lambda_{S O} \simeq 0.2 t$. We have selected strong enough magnetic coupling $J=6 t$ which allows for the QPT driven by $\lambda_{S O}$. The upper panel of Fig. 5 displays the bulk polarization, defined as

$$
\mathcal{P}=\frac{1}{2} \sum_{\mathbf{i}}\left(\left\langle n_{\mathbf{i} \uparrow}\right\rangle-\left\langle n_{\mathbf{i} \downarrow}\right\rangle\right)
$$

where $n_{\mathbf{i} \sigma}=\sum_{n}^{\prime}\left[\left|u_{\mathbf{i} \sigma}^{n}\right|^{2} f\left(E_{n}, T\right)+\left|v_{\mathbf{i} \sigma}^{n}\right|^{2} f\left(-E_{n}, T\right)\right]$ is the average number of electrons with spin $\sigma$ at site $\mathbf{i}$, the order parameter at the impurity site, and its value averaged over the entire sample. At $\lambda_{S O} \approx 0.05 t$ the order parameter at impurity site reverses its sign and its absolute value gradually increases upon increasing the KaneMele coupling. Simultaneously the bulk magnetization is abruptly quenched as the system shifts to the unpolarized ground state. These characteristic features of the $\mathrm{QPT}^{1}$ in the present case originate from the intrinsic SOC. On the other hand, the bulk order parameter does not undergo any dramatic changes (it slowly diminishes upon increasing $\lambda_{S O}$ ). Such conditions should be taken into account when considering formation of the Majorana bound states at the ends of magnetic chains deposited on the proximitized honeycomb sheet ${ }^{25}$.

The shift of $J_{c}$ with increasing $\lambda_{S O}$ is displayed as a phase diagram in Fig. 6 . The black continuous line denotes the critical coupling $J_{c}$ at different values of $\lambda_{S O}$. Initially the shift of QPT is not meaningful, but starting from $\lambda_{S O}=0.03 t$ we observe onset of a linear variation. This increase also points out, that the spin-orbit interaction supresses the effective coupling of the impurity spin with the conduction electrons ${ }^{36}$. 


\section{Topography of Shiba quasiparticles}

Let us now inspect the real-space shape (topography) of the Shiba states. Fig. 7 presents spatial maps of the density of states at the energy of electron-like (occupied) bound state, both in absence and in presence of the intrinsic spin-orbit interaction. One can see that without the Kane-Mele interaction, the topography of Shiba state has its usual character with exponential variation of the wavefuction $\sim \exp (-r / \xi)$. We remark, that spectral weight is differently distributed in each sublattice. Close to the impurity site $r_{0}=(0,0)$ most of the spectral weight of the Shiba quasiparticles appears in the B-sublattice sites, whereas further away the A-sublattice (in which the impurity resides) gains more and more spectral weight. Also the rotational symmetry of the topographic shape reveals a bipartite character. Close to the impurity site the shape has a $C_{3}$ rotational symmetry, reflecting the fact that each site has three nearest-neighbors (cf. bottom panels in Fig. 2), whereas at larger distances, the spectral weight distributed in the A sublattice resembles a hexagon with $C_{6}$ rotational symmetry. Precise evaluation of the bound states wavefuntions in this case would be a challenging task for future experimental measurements. Topography of the bound states changes dramatically, when the intrinsic SOC is taken into account. Bottom panel in Fig. 7 illustrates a strong tendency towards localization of the Shiba states in vicinity of the magnetic impurity. Their spectral weight is spread over a few adjacent sites and we no longer observe any preference for dominance of only one sublattice. These properties of the Shiba states resemble the features typical for in-gap quasiparticles of magnetic impurity embedded in a non-superconducting QSHI. Such reduction of the spatial extent could be important for engineering the topologically non-trivial phases, as e.g. chain of magnetic impurities can host the Majorana quasiparticles only when the bound states of dilute impurities hybrydize to form a Shiba band capable of undergoing the topological phase transition.
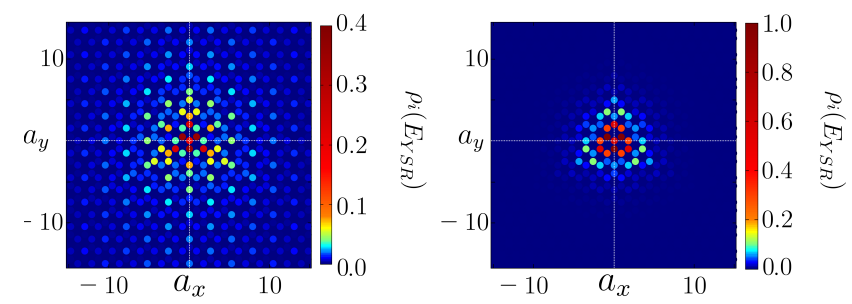

FIG. 7. Spatial distribution of the occupied (negative value) Shiba quasiparticle obtained for $\Delta=0.2 t, \lambda_{R}=0.05 t, J=4 t$, using $\lambda_{S O}=0$ (left panel) and $\lambda_{S O}=0.1 t$ (right panel). The density of states $\rho_{i}\left(E_{\text {Shiba }}\right)$ is normalized with respect to the largest value in the bottom panel.

\section{E. Local currents}

Another signature of the QPT in our system can be seen by currents induced around the magnetic impurity ${ }^{53}$. We compute the local charge flow, using the Heisenberg equation $i \hbar \frac{\partial\left\langle n_{i}\right\rangle}{\partial t}=\left\langle\left[n_{i}, \hat{H}\right]\right\rangle$. Setting the convention $\hbar \equiv 1$, and ignoring the terms which merely induce on-site fluctuations of charge, we obtain

$$
\begin{aligned}
& \frac{\partial\left\langle n_{\mathbf{i}}\right\rangle}{\partial t}=-i t \sum_{\sigma\langle\mathbf{j}\rangle}\left(\left\langle c_{\mathbf{i} \sigma}^{\dagger} c_{\mathbf{j} \sigma}\right\rangle-\left\langle c_{\mathbf{j} \sigma}^{\dagger} c_{\mathbf{i} \sigma}\right\rangle\right) \\
+ & \lambda_{S O} \sum_{\sigma \sigma^{\prime}\langle\langle\mathbf{j}\rangle\rangle}\left(\nu_{\mathbf{i j}} s_{z}^{\sigma \sigma^{\prime}}\left\langle c_{\mathbf{i} \sigma^{\prime}}^{\dagger} c_{\mathbf{j} \sigma}\right\rangle-\nu_{\mathbf{j i}} s_{z}^{\sigma^{\prime} \sigma}\left\langle c_{\mathbf{j} \sigma^{\prime}}^{\dagger} c_{\mathbf{i} \sigma}\right\rangle\right) \\
+ & \lambda_{R} \sum_{\sigma \sigma^{\prime}\langle\mathbf{j}\rangle}\left[\left(\mathbf{s}^{\sigma \sigma^{\prime}} \times \mathbf{d}_{\mathbf{i j}}\right)_{\vec{z}}\left\langle c_{\mathbf{i} \sigma}^{\dagger} c_{\mathbf{j} \sigma^{\prime}}\right\rangle-\left(\mathbf{s}^{\sigma^{\prime} \sigma} \times \mathbf{d}_{\mathbf{j i}}\right)_{\vec{z}}\left\langle c_{\mathbf{j} \sigma^{\prime}}^{\dagger} c_{\mathbf{i} \sigma}\right\rangle\right] .
\end{aligned}
$$

Applying the Bogoliubov-Valatin transformation (6), and making use of the fact that if $\Phi_{\mathbf{i}}=\left(u_{\mathbf{i} \uparrow}^{n}, u_{\mathbf{i} \downarrow}^{n}, v_{\mathbf{i} \uparrow}^{n}, v_{\mathbf{i} \downarrow}^{n}\right)^{T}$ is the eigenvector of matrix (8) with an eigenvalue $E_{n}$, then $\tilde{\Phi}_{\mathbf{i}}=\left(-v_{\mathbf{i} \uparrow}^{n *}, v_{\mathbf{i} \downarrow}^{n *},-u_{\mathbf{i} \uparrow}^{n *}, u_{\mathbf{i} \downarrow}^{n *}\right)^{T}$ is also an eigenvector of the same matrix, but with an eigenvalue $-E_{n}$, we get

$$
\begin{aligned}
\left\langle\hat{J}_{\mathbf{i}}\right\rangle & =-i t \sum_{\langle\mathbf{j}\rangle \sigma n}\left(u_{\mathbf{i} \sigma}^{n *} u_{\mathbf{j} \sigma}^{n} f_{F D}\left(E_{n}\right)-\text { c.c. }\right) \\
& +\sum_{\langle\mathbf{j}\rangle \sigma \sigma^{\prime} n}\left(\lambda_{R}^{\sigma \sigma^{\prime}} u_{\mathbf{i} \sigma}^{n *} u_{\mathbf{j} \sigma^{\prime}}^{n} f_{F D}\left(E_{n}\right)+\text { c.c. }\right) \\
& +\lambda_{S O} \sum_{\langle\langle\mathbf{j}\rangle\rangle \sigma \sigma^{\prime} n}\left(\nu_{\mathbf{i} \mathbf{j}} s_{z}^{\sigma \sigma^{\prime}} u_{\mathbf{i} \sigma}^{n *} u_{\mathbf{j} \sigma^{\prime}}^{n} f_{F D}\left(E_{n}\right)+\text { c.c. }\right)
\end{aligned}
$$

where $\lambda_{R}^{\sigma \sigma^{\prime}}$ defined in Sec. II.

Fig. 8 shows the real-space maps of microscopic currents and the maximum magnitude of bond current in the system with respect to the impurity coupling strength $J$. We emphasize, that reversal of these currents vorticity (compare the top panels of Fig. 8) occurs exclusively when the system is in the non-trivial QSHI phase. Explanation of this behavior could be the following. It has been shown in Ref. ${ }^{36}$ that the intrinsic SOC supresses the effective coupling $J$ of impurity with the conduction electrons. We have observed that with $\lambda_{S O}=0$ the sites belonging to the same sublattice as the impurity site polarize easily in the direction of the magnetic moment of the impurity. This effect is pronounced only for $J>J_{C}$, as more sites around the impurity align their magnetic moments. The situation changes with increasing SOC which weakens the effective impurity coupling. For small $J$, the magnetic moment is hardly screened by the closest neighboring sites and becomes more efficient when the coupling exceeds the critical value $J_{C}$, forcing the neighboring sites to align their magnetization with the impurity. This in turn reverses the direction of the current. The Shiba states (labeled YSR in red vector plots in Fig. 8) are the ones that cross the Fermi energy during the QPT, hence only their contribution to the current shows this change of direction, in contrast to the 


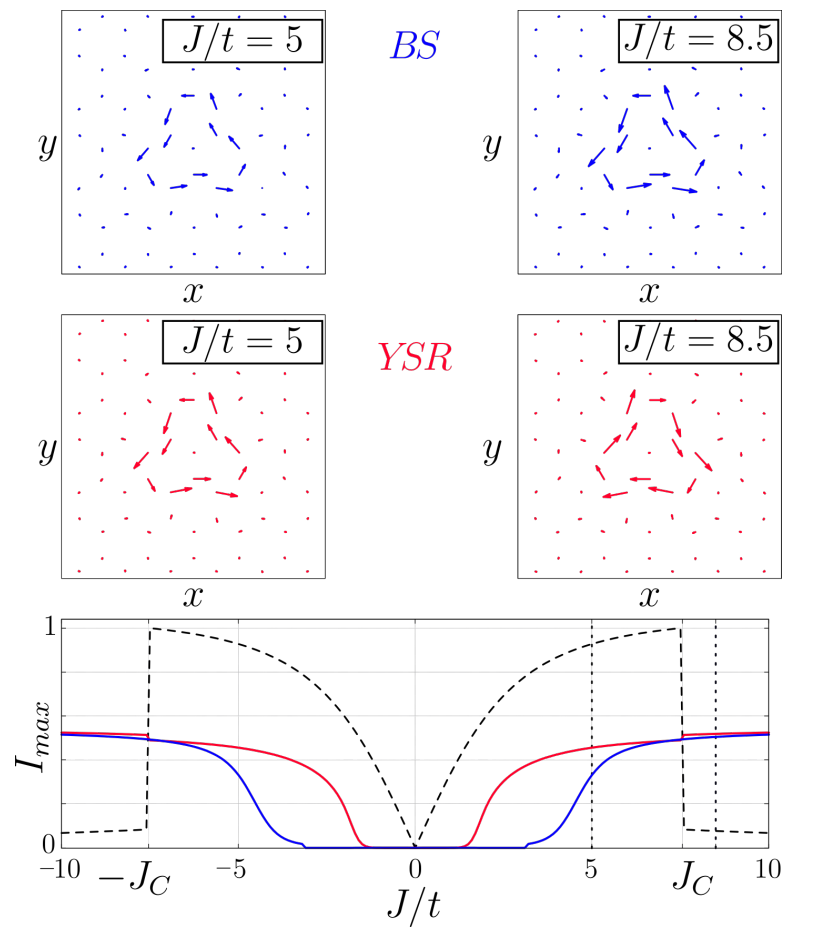

FIG. 8. Vector maps of the currents around the magnetic impurity obtained for $J=5 t$ (left) and $J=8.5 t$ (right) presenting cotributions of QSHI bound states (BS) and Shiba states (YSR). Bottom panel shows the maximum current versus the coupling $J$ when taking into account the whole spectrum (black dashed line), only QSHI bound states and Shiba states (blue and red lines respectively). Other parameters: $\Delta=0.2 t, \lambda_{S O}=0.1 t, \lambda_{R}=0.05 t$.

bound states (BS in blue vector plots in Fig. 8) discussed in Sec. III A. Those states hardly change their energy with incresing $J$, and their contribution to the current does not change during the QPT. Bottom panel in Fig. 8 presents the maximum value of the current in the system. When summing over every state $n$ in Eq. 11, the current drops discontinuously at QPT. This is because as can be observed from the contribution of only the Shiba states (red) and the QSHI bound states (blue), after the reversal of current direction of the Shiba states, both contributions compete, and the effective maximum current is greatly reduced. Detection of such orbital patterns might be performed using an integrated quantum imaging platform where graphene sheet is connected to an array of the atomic-sized magnetic sensors ${ }^{54,55}$ or local conductivity atomic force microscopy suitable for probing electronic current paths with a diameter in the nanometer range ${ }^{56}$.

\section{CONCLUSIONS}

We have theoretically investigated the energetic, magnetic and topographic features of in-gap quasiparticles of a single magnetic impurity embedded in the graphene sheet and proximitized to the superconducting substrate. We have shown that subtle interplay between the intrinsic spin-orbit interaction (responsible for the energy gap of the QSHI phase) and the proximity-induced electron pairing enables emergence of the Shiba-type quasiparticles directly from in-gap states of the insulating phase. We have discussed in detail this intriguing behavior and proposed several methods for its empirical verification.

Furthermore we have found, that upon varying either the magnetic coupling $J$ (feasible in STM experiments ${ }^{18}$ ) or strength of the spin-orbit coupling $\lambda_{S O}$ a pair of the Shiba bound states could cross at the Fermi energy, causing quantum phase transition of the ground state. This usually leads to sign-change of the local order parameter ${ }^{1}$, but in the present situation it would be also uniquely manifested by a reversal of the vorticity and abrupt change of the absolute magnitude of the local currents circulating around the impurity site. Our numerical calculations have additionally revealed, that the spinorbit coupling pushes such QPT crossing towards much higher values of $J$ and substantially reduces the extent of Shiba states (similar to the in-gap states of insulating phase), partly affecting their topographic patterns. We have carefully inspected their spatial profiles in each sublattice of the graphene sheet.

We hope that phenomena discussed here for the singlesite magnetic defects ${ }^{57,58}$ might stimulate further considerations of the topological insulating and/or superconducting phases in more complex magnetic structures, like nanowires ${ }^{24,25}$, nanoscopic islands ${ }^{19}$ or stripes ${ }^{26,27}$, where the Majorana-type quasiparticles can be realized. It would be also worth to extend our study on the quantum impurities, addressing the subgap Kondo physics of the conventional ${ }^{59,60}$ and topological ${ }^{61}$ superconductors.

\section{ACKNOWLEDGMENTS}

This work was supported by National Science Centre (NCN, Poland) under the grants 2017/27/N/ST3/01762 (S.G.) and UMO-2017/27/B/ST3/01911 (T.D.).
* e-mail: szglodzik@kft.umcs.lublin.pl

† e-mail: doman@kft.umcs.lublin.pl

1 A. V. Balatsky, I. Vekhter, and J.-X. Zhu, "Impurityinduced states in conventional and unconventional superconductors," Rev. Mod. Phys. 78, 373 (2006).

2 B.W. Heinrich, J.I. Pascual, and K.J. Franke, "Single mag- netic adsorbates on s-wave superconductors," Prog. Surf. Science 93, 1 (2018).

3 C. L. Kane and E. J. Mele, "Quantum spin Hall effect in graphene," Phys. Rev. Lett. 95, 226801 (2005).

4 K. Hatsuda, H. Mine, T. Nakamura, J. Li, R. Wu, S. Katsumoto, and J. Haruyama, "Evidence for a quantum spin 
hall phase in graphene decorated with $\mathrm{Bi}_{2} \mathrm{Te}_{3}$ nanoparticles," Science Advances 4 (2018), 10.1126/sciadv.aau6915.

${ }^{5}$ S. Wu, V. Fatemi, Q.D. Gibson, K. Watanabe, T. Taniguchi, R.J. Cava, and P. Jarillo-Herrero, "Observation of the quantum spin Hall effect up to 100 kelvin in a monolayer crystal," Science 359, 76-79 (2018).

${ }^{6}$ H. B. Heersche, P. Jarillo-Herrero, J. B. Oostinga, L. M. K. Vandersypen, and A. F. Morpurgo, "Bipolar supercurrent in graphene," Nature 446 (2007), 10.1038/nature05555.

7 K. Komatsu, Ch. Li, S. Autier-Laurent, H. Bouchiat, and S. Guéron, "Superconducting proximity effect in long superconductor/graphene/superconductor junctions: From specular Andreev reflection at zero field to the quantum Hall regime," Phys. Rev. B 86, 115412 (2012).

8 C. Tonnoir, A. Kimouche, J. Coraux, L. Magaud, B. Delsol, B. Gilles, and C. Chapelier, "Induced superconductivity in graphene grown on rhenium," Phys. Rev. Lett. 111, 246805 (2013).

9 Z. Han, A. Allain, H. Arjmandi-Tash, K. Tikhonov, M. Feigelman, B. Sacépé, and V. Bouchiat, "Collapse of superconductivity in a hybrid tingraphene Josephson junction array," Nature Phys. 10, 380 (2014).

10 V. E. Calado, S. Goswami, G. Nanda, M. Diez, A. R. Akhmerov, K. Watanabe, T. Taniguchi, T. M. Klapwijk, and L. M. K. Vandersypen, "Ballistic Josephson junctions in edge-contacted graphene," Nature Nanotech. 10, 761 (2015).

11 Fabian D. Natterer, Jeonghoon Ha, Hongwoo Baek, Duming Zhang, William G. Cullen, Nikolai B. Zhitenev, Young Kuk, and Joseph A. Stroscio, "Scanning tunneling spectroscopy of proximity superconductivity in epitaxial multilayer graphene," Phys. Rev. B 93, 045406 (2016).

12 J. W. González and J. Fernández-Rossier, "Impurity states in the quantum spin Hall phase in graphene," Phys. Rev. B 86, 115327 (2012).

13 Y. Cao, V. Fatemi, S. Fang, K. Watanabe, T. Taniguchi, E. Kaxiras, and P. Jarillo-Herrero, "Unconventional superconductivity in magic-angle graphene superlattices," Nature 556, 43 (2018).

14 X. Lu, P. Stepanov, W. Yang, M.A. Xie, M. Aamir, I. Das, C. Urgell, K. Watanabe, T. Taniguchi, G. Zhang, A. Bachtold, A.H. MacDonald, and D.K. Efetov, "Superconductors, orbital magnets and correlated states in magic-angle bilayer graphene," Nature 574, 653 (2019).

15 M. Yankowitz, S. Chen, H. Polshyn, Y. Zhang, K. Watanabe, T. Taniguchi, D. Graf, A.F. Young, and C.R. Dean, "Tuning superconductivity in twisted bilayer graphene," Science 363, 1059 (2019), https://science.sciencemag.org/content/363/6431/1059.full.pdï.

16 G. C. Ménard, S. Guissart, Ch. Brun, S. Pons, V. S. Stolyarov, F. Debontridder, M. V. Leclerc, E. Janod, L. Cario, D. Roditchev, P. Simon, and T. Cren, "Coherent longrange magnetic bound states in a superconductor," Nature Physics 11, 1013 (2015).

17 N. Hatter, B. W. Heinrich, D. Rolf, and K. J. Franke, "Scaling of $\mathrm{Yu}$-Shiba-Rusinov energies in the weakcoupling Kondo regime," Nature Comm. 8, 2016 (2017).

${ }^{18}$ L. Farinacci, G. Ahmadi, G. Reecht, M. Ruby, N. Bogdanoff, O. Peters, B. W. Heinrich, F. von Oppen, and K. J. Franke, "Tuning the coupling of an individual magnetic impurity to a superconductor: Quantum phase transition and transport," Phys. Rev. Lett. 121, 196803 (2018).

19 G. C. Ménard, C. Brun, R. Leriche, M. Trif, F. Debontridder, D. Demaille, D. Roditchev, P. Simon, and T. Cren,
"Yu-Shiba-Rusinov bound states versus topological edge states in $\mathrm{Pb} / \mathrm{Si}(111)$," Eur. Phys. J. Special Topics (2019), 10.1140/epjst/e2018-800056-3.

20 S. Kezilebieke, M. Dvorak, T. Ojanen, and P. Liljeroth, "Coupled Yu-Shiba-Rusinov states in molecular dimers on $\mathrm{NbSe}_{2}$," Nano Letters 18, 2311-2315 (2018).

21 A. Ptok, S. Głodzik, and T. Domański, "Yu-ShibaRusinov states of impurities in a triangular lattice of $\mathrm{NbSe}_{2}$ with spin-orbit coupling," Phys. Rev. B 96, 184425 (2017).

22 S. Körber, B. Trauzettel, and O. Kashuba, "Collective Yu-Shiba-Rusinov states in magnetic clusters at superconducting surfaces," Phys. Rev. B 97, 184503 (2018).

23 J. Senkpiel, C. Rubio-Verdú, M. Etzkorn, R. Drost, L.M. Schoop, S. Dambach, C. Padurariu, B. Kubala, J. Ankerhold, Ch.R. Ast, and K. Kern, "Robustness of Yu-ShibaRusinov resonances in the presence of a complex superconducting order parameter," Phys. Rev. B 100, 014502 (2019).

24 M.H. Christensen, M. Schecter, K. Flensberg, B.M. Andersen, and J. Paaske, "Spiral magnetic order and topological superconductivity in a chain of magnetic adatoms on a two-dimensional superconductor," Phys. Rev. B 94, 144509 (2016).

25 R. L. R. C. Teixeira, D. Kuzmanovski, A. M. BlackSchaffer, and L. G. G. V. Dias da Silva, "Gap oscillations and Majorana bound states in magnetic chains on superconducting honeycomb lattices," Phys. Rev. B 99, 035127 (2019).

26 A. Fornieri, A.M. Whiticar, F. Setiawan, E. Portoles, A.C.C. Drachmann, A. Keselman, S. Gronin, C. Thomas, T. Wang, R. Kallaher, G.C. Gardner, E. Berg, M.J. Manfra, A. Stern, Ch.M. Marcus, and F. Nichele, "Evidence of topological superconductivity in planar Josephson junctions," Nature 569, 89 (2019).

27 H. Ren, F. Pientka, S. Hart, A.T. Pierce, M. Kosowsky, L. Lunczer, R. Schlereth, B. Scharf, E.M. Hankiewicz, L.W. Molenkamp, B.I. Halperin, and A. Yacoby, "Topological superconductivity in a phase-controlled Josephson junction," Nature 569, 93 (2019).

28 J. Sichau, M. Prada, T. Anlauf, T. J. Lyon, B. Bosnjak, L. Tiemann, and R. H. Blick, "Resonance microwave measurements of an intrinsic spin-orbit coupling gap in graphene: A possible indication of a topological state," Phys. Rev. Lett. 122, 046403 (2019).

29 T. Meng, J. Klinovaja, S. Hoffman, P. Simon, and D. Loss, "Superconducting gap renormalization around two magnetic impurities: From Shiba to Andreev bound states," Phys. Rev. B 92, 064503 (2015).

. S. Hoffman, J. Klinovaja, T. Meng, and D. Loss, "Impurity-induced quantum phase transitions and magnetic order in conventional superconductors: Competition between bound and quasiparticle states," Phys. Rev. B 92, 125422 (2015).

31 A. M. Black-Schaffer, "Self-consistent superconducting proximity effect at the quantum spin Hall edge," Phys. Rev. B 83, 060504 (2011).

32 J. H. Zheng and M. A. Cazalilla, "Nontrivial interplay of strong disorder and interactions in quantum spin-Hall insulators doped with dilute magnetic impurities," Phys. Rev. B 97, 235402 (2018).

33 R. J. Slager, L. Rademaker, J. Zaanen, and L. Balents, "Impurity-bound states and Green's function zeros as local signatures of topology," Phys. Rev. B 92, 085126 (2015).

34 J. Maciejko, C. Liu, Y. Oreg, X.-L. Qi, C. Wu, and S.- 
C. Zhang, "Kondo effect in the helical edge liquid of the quantum spin Hall state," Phys. Rev. Lett. 102, 256803 (2009).

35 F. Goth, D. J. Luitz, and F. F. Assaad, "Magnetic impurities in the Kane-Mele model," Phys. Rev. B 88, 075110 (2013).

36 F. M. Hu, T. O. Wehling, J. E. Gubernatis, Thomas Frauenheim, and R. M. Nieminen, "Magnetic impurity affected by spin-orbit coupling: Behavior near a topological phase transition," Phys. Rev. B 88, 045106 (2013).

37 Q. Liu, C.-X. Liu, C. Xu, X.-L. Qi, and S.-C. Zhang, "Magnetic impurities on the surface of a topological insulator," Phys. Rev. Lett. 102, 156603 (2009).

38 A. A. Zyuzin and D. Loss, "RKKY interaction on surfaces of topological insulators with superconducting proximity effect," Phys. Rev. B 90, 125443 (2014).

39 H.-H. Lee, J.-Y. Liu, C.-R. Chang, and S.-Q. Shen, "Impurity influence in quantum spin Hall transport," Phys. Rev. B 88, 195149 (2013).

40 A. M. Black-Schaffer and A. V. Balatsky, "Strong potential impurities on the surface of a topological insulator," Phys. Rev. B 85, 121103 (2012).

41 P. Sessi, R. R. Biswas, T. Bathon, O. Storz, S. Wilfert, A. Barla, K. A. Kokh, O. E. Tereshchenko, K. Fauth, M. Bode, and A. V. Balatsky, "Dual nature of magnetic dopants and competing trends in topological insulators," Nature Commun. 7, 12027 (2016), article.

42 R. R. Biswas and A. V. Balatsky, "Impurity-induced states on the surface of three-dimensional topological insulators," Phys. Rev. B 81, 233405 (2010).

43 A. M. Black-Schaffer, A. V. Balatsky, and J. Fransson, "Filling of magnetic-impurity-induced gap in topological insulators by potential scattering," Phys. Rev. B 91, 201411 (2015).

44 P. Nozières and F. Pistolesi, "From semiconductors to superconductors: a simple model for pseudogaps," Eur. Phys. J. B 10, 649-662 (1999).

45 Y. L. Loh, M. Randeria, N. Trivedi, C.-C. Chang, and R. Scalettar, "Superconductor-insulator transition and Fermi-Bose crossovers," Phys. Rev. X 6, 021029 (2016).

46 A. Yazdani and A. Kapitulnik, "Superconductinginsulating transition in two-dimensional $a$-MoGe thin films," Phys. Rev. Lett. 74, 3037 (1995).

47 A. D. Caviglia, S. Gariglio, N. Reyren, D. Jaccard, T. Schneider, M. Gabay, S. Thiel, G. Hammerl, J. Mannhart, and J.-M. Triscone, "Electric field control of the $\mathrm{LaAlO}_{3} / \mathrm{SrTiO}_{3}$ interface ground state," Nature (London) 456, 624-627 (2008).
${ }^{48}$ K. Kanoda and R. Kato, "Mott physics in organic conductors with triangular lattices," Annu. Rev. Condens. Matter Phys. 2, 167-188 (2011).

49 A. T. Bollinger, G. Dubuis, J. Yoon, D. Pavuna, J. Misewich, and I. Bozovic, "Superconductorinsulator transition in $\mathrm{La}_{2-x} \mathrm{Sr}_{x} \mathrm{CuO}_{4}$ at the pair quantum resistance," Nature (London) 472, 458-460 (2011).

50 J. L. Lado and J. Fernéndez-Rossier, "Unconventional YuShiba-Rusinov states in hydrogenated graphene," 2D Materials 3, 025001 (2016).

51 T. O. Wehling, H. P. Dahal, A. I. Lichtenstein, and A. V. Balatsky, "Local impurity effects in superconducting graphene," Phys. Rev. B 78, 035414 (2008).

52 A. Sakurai, "Comments on superconductors with magnetic impurities," Prog. Theor. Phys. 44, 1472 (1970).

53 S. S. Pershoguba, K. Björnson, A. M. Black-Schaffer, and A. V. Balatsky, "Currents induced by magnetic impurities in superconductors with spin-orbit coupling," Phys. Rev. Lett. 115, 116602 (2015).

54 J.-Ph. Tetienne, N. Dontschuk, D.A. Broadway, A. Stacey, D.A. Simpson, and L.C.L. Hollenberg, "Quantum imaging of current flow in graphene," Science Adv. 3 (2017), $10.1126 /$ sciadv. 1602429 .

55 Francesco Casola, Toeno van der Sar, and Amir Yacoby, "Probing condensed matter physics with magnetometry based on nitrogen-vacancy centres in diamond," Nature Reviews Materials 3, 17088 (2018).

56 C. Rodenbücher, G. Bihlmayer, W. Speier, J. Kubacki, M. Wojtyniak, M. Rogala, D. Wrana, F. Krok, and K. Szot, "Local surface conductivity of transition metal oxides mapped with true atomic resolution," Nanoscale 10, 11498 (2018).

57 O.V. Yazyev and L. Helm, "Defect-induced magnetism in graphene," Phys. Rev. B 75, 125408 (2007).

58 Alejandro Lopez-Bezanilla and J. L. Lado, "Defect-induced magnetism and Yu-Shiba-Rusinov states in twisted bilayer graphene," Phys. Rev. Materials 3, 084003 (2019).

59 J. Bauer, A. Oguri, and A.C. Hewson, "Spectral properties of locally correlated electrons in a Bardeen-Cooper-Schrieffer superconductor," J. Phys.: Condens. Matter 19, 486211 (2007).

60 C. Liu, Y. Huang, Y. Chen, and C.S. Ting, "Temperaturedependent spectral function of a Kondo impurity in an $s$ wave superconductor," Phys. Rev. B 99, 174502 (2019).

61 R. Wang, W. Su, J.-X. Zhu, C.S. Ting, H. Li, C. Chen, B. Wang, and X. Wang, "Kondo signatures of a quantum magnetic impurity in topological superconductors," Phys. Rev. Lett. 122, 087001 (2019). 